\title{
Restoration of fibroblast growth factor receptor 2 IIIb enhances the chemosensitivity of human prostate cancer cells
}

\author{
KOICHI SHOJI ${ }^{1}$, JUN TEISHIMA ${ }^{1}$, TETSUTARO HAYASHI ${ }^{1}$, SHINYA OHARA ${ }^{1}$, \\ WALLACE L. McKEEHAN ${ }^{2}$ and AKIO MATSUBARA ${ }^{1}$ \\ ${ }^{1}$ Department of Urology, Institute of Biomedical and Health Sciences, Integrated Health Science, \\ Hiroshima University, Hiroshima 734-8551, Japan; ${ }^{2}$ Center for Cancer and Stem Cell Biology, \\ Institute of Bioscience and Technology, Texas A\&M Health Science Center, Houston, TX 77030, USA
}

Received February 5, 2014; Accepted April 23, 2014

DOI: $10.3892 / o r .2014 .3200$

\begin{abstract}
Fibroblast growth factor receptor 2 (FGFR2) is thought to mediate an important signaling pathway between prostate epithelial cells and stromal cells for maintenance of homeostasis in normal prostate tissue. Abnormalities of FGFR2 have been shown in advanced prostate cancer or prostate cancer cell lines, and we previously demonstrated the tumor-suppressive effects of the restoration of FGFR2IIIb in prostate cancer cells. The aim of the present study was to determine whether FGFR2IIIb plays a role in the chemosensitivity of castration-resistant prostate cancer cells. A clonal line of PC-3 cells expressing FGFR2IIIb (PC-3R2IIIb) was established by transfection with an IRESneo2-expressing vector bearing FGFR2IIIb cDNA. The effects of chemotherapeutic agents (docetaxel, cisplatin, 5-fluorouracil and zoledronic acid) on cell viability and apoptosis were examined by MTT assay and western blot analysis, respectively. Expression levels of molecules that were markers of epithelial-to-mesenchymal transition and chemosensitivity-related proteins were assessed by western blot analysis. Viability of the PC-3R2IIIb cells was significantly lower than that of the control PC-3 cells transfected with the vector alone (PC-3neo), and viability was further suppressed by treatment with chemotherapeutic agents, particularly docetaxel. Induced expression of caspase-3 was evident in the PC-3R2IIIb cells and was further enhanced by treatment with docetaxel. Expression of $\mathrm{N}$-cadherin, vimentin, survivin and XIAP was lower in the PC-3R2IIIb cells than that in the PC-3neo cells. In contrast, expression of p21 was higher in the PC-3R2IIIb cells than that in the control PC-3neo cells. These data indicate that restoration of FGFR2IIIb in
\end{abstract}

Correspondence to: Dr Jun Teishima, Department of Urology, Institute of Biomedical and Health Sciences, Integrated Health Science, Hiroshima University, 1-2-3 Kasumi, Minami-ku, Hiroshima 734-8551, Japan

E-mail: teishima@hiroshima-u.ac.jp

Key words: fibroblast growth factor receptor 2IIIb, prostate cancer, docetaxel, chemosensitivity castration-resistant prostate cancer cells may reverse some of the epithelial-to-mesenchymal cell properties characteristic of tumor cells and induce in part mesenchymal-to-epithelial transition properties. This together with enhancement of apoptotic pathways involving caspase- 3 may enhance chemosensitivity particularly to docetaxel which is widely used in the treatment of castration-resistant prostate cancer.

\section{Introduction}

Prostate cancer is one of the most common malignant neoplasms among men and is the second leading cause of male cancer-related death in the United States (1-3). Most prostate cancers initially show characteristics of androgen-dependent growth and are sensitive to androgen deprivation therapy (ADT). However, these cancers eventually acquire the ability of castration-resistant growth and become resistant to ADT (4). Although chemotherapy that includes docetaxel has been regarded as an effective choice in the clinical strategy against castration-resistant prostate cancer (CRPC), its anticancer effect alone appears insufficient (5). Eventually, resistance to chemotherapy develops, and disease control becomes even more difficult. Clarification of molecular mechanisms in CRPC for development of novel therapeutic strategies against this resistant cancer remains a high priority.

Fibroblast growth factor (FGF) signaling has been implicated in cellular homeostasis in normal prostate tissue by mediating communication between epithelial and stromal cells (6-8). FGF receptor type 2IIIb (FGFR2IIIb) is one of the splicing variants of FGFR2 generally associated with differentiated epithelial cells and is expressed in prostate epithelial cells. It is specific for FGF7 (keratinocyte growth factor, KGF) and FGF10, which when expressed are associated with stromal cells (6,9-16). Prostate stromal cells secrete FGF7 or FGF10 in response to androgen stimulation, and signals elicited by their activation of FGFR2IIIb have been correlated with cell growth, cell differentiation or apoptosis $(6,12,16-18)$. The loss or abnormalities of FGFR2IIIb expression have been demonstrated in prostate cancer, especially in progressive or castration-resistant cancers (15-21). Previous studies have shown that restoration of FGFR2IIIb in prostate cancer cells leads to suppression of cell growth through KGF-FGFR2IIIb 
signaling, induction of apoptosis and generally tumorsuppressive effects in vivo $(18,19)$. These reports suggest that disorders of the KGF-FGFR2IIIb signaling pathway may be an important event for CRPC to acquire greater growth potential and tumorigenic properties. This suggests that the manipulation of FGFR2IIIb or its tumor-suppressive pathways deserves attention as a novel therapeutic target. In addition to this evidence of the effects of the KGF-FGFR2IIIb signaling pathway, several studies have shown the involvement of the FGF system in chemoresistance in malignant cells (22). In the present study, we investigated the effects of the restoration of FGFR2IIIb on the chemosensitivity of CRPC cells as a guide for possible development of new therapeutic strategies and biomarkers of their efficacy for CRPC.

\section{Materials and methods}

Cell culture and transfections. The PC-3 cell line is one of the representative prostate cancer cell lines with the ability for castration-resistant growth (23). Several studies have shown its unresponsiveness to hormones and resistance to apoptosis (23-25). In PC-3 cells, abnormalities of FGF and FGFR expression including the loss of FGFR2IIIb expression have been demonstrated (18). For these reasons, we used PC-3 cells in the present study.

Cloned PC-3 cells were maintained in OPTI-MEM1 medium (Life Technologies, Carlsbad, CA, USA) supplemented with $5 \%$ heat-inactivated fetal bovine serum (FBS; Life Technologies) and $100 \mathrm{mg} / \mathrm{ml}$ kanamycin (Life Technologies). Preparation of FGFR2IIIb cDNA and transfection to PC-3 cells have been previously described (18). Briefly, full-length FGFR2IIIb cDNA was cloned into the IRESneo2 expression vector (Clontech Laboratories, Inc., Palo Alto, CA, USA) (IRESneo2-FGF2IIIb). PC-3 cells were transfected with IRESneo2-FGFR2IIIb or the IRESneo2 empty control vector by electroporation using the Gene Pulser II apparatus (Bio-Rad Laboratories, Hercules, CA, USA). The cells were suspended and incubated in OPTI-MEM1 medium supplemented with $5 \%$ heat-inactivated FBS and $100 \mathrm{mg} / \mathrm{ml}$ kanamycin containing $200 \mathrm{mg} / \mathrm{ml} \mathrm{G} 418$ sulfate (Promega, Madison, WI, USA) for 14 days. Colonies of cells were picked up and maintained in medium containing $200 \mathrm{mg} / \mathrm{ml}$ of G418 sulfate. Expression of FGFR2IIIb was analyzed by western immunoblotting with mouse anti-FGFR2IIIb antibody (Santa Cruz Biotechnology, Santa Cruz, CA, USA). PC-3 cell lines transfected with FGFR2IIIb were designated as PC-3R2IIIb, and control cell cultures transfected with empty vector were designated as PC-3neo.

Assessment of cell viability. Cell viability and indirectly cell numbers were measured with the MTT assay. PC-3R2IIIb and PC-3neo cells (1,000 cells/well) were each plated in 96-well plates containing $100 \mu \mathrm{l}$ of OPTI-MEM1 medium and 5\% heat-inactivated FBS and incubated at $37^{\circ} \mathrm{C}$ under $5 \% \mathrm{CO}_{2}$ overnight. To determine the cytotoxic effect of chemotherapeutic agents, cells were treated with a range of concentrations of docetaxel, cisplatin, 5-fluorouracil or zoledronic acid. After $1 \mathrm{~h}$, cells in each well were washed with phosphate-buffered saline (PBS) and incubated in $100 \mu$ of OPTI-MEM1 medium plus 5\% heat-inactivated FBS. On days 2 and 4, the MTT solu- tion was added followed by incubation at $37^{\circ} \mathrm{C}$ for $4 \mathrm{~h}$. Cell viability was measured using an ELISA plate reader (Bio-Rad Laboratories) at $570 \mathrm{~nm}$ with the reference wavelength of $630 \mathrm{~nm}$.

Assessment of biomarker expression. Western blot analysis was performed essentially as previously described (18). The following antibodies were used at dilutions of 1:1,000: anticaspase-3 rabbit monoclonal antibody (\#9662; Cell Signaling Technology, Beverly, MA, USA), anti-p53 mouse monoclonal antibody (\#2015; Cell Signaling Technology), anti-p21 rabbit monoclonal antibody (ab7960; Abcam, Cambridge, MA, USA), anti-N-cadherin rabbit monoclonal antibody (ab1221; Abcam), anti-E-cadherin rabbit monoclonal antibody (ab53033; Abcam), anti-vimentin mouse monoclonal antibody (ab115191; Abcam), anti-MDR1 monoclonal antibody (clone C219; Calbiochem, Darmstadt, Germany), anti-clusterin rabbit polyclonal antibody (GTX101236; GeneTex, Irvine, CA, USA), anti-Bcl-2 rabbit polyclonal antibody (GTX101291; GeneTex), anti-Bcl2-like 1 rabbit polyclonal antibody (GTX105661; GeneTex), antiMdm2 p53 binding protein homolog (mouse) rabbit polyclonal antibody (GTX100654; GeneTex), anti-X-linked inhibitor of apoptosis rabbit polyclonal antibody (GTX113130; GeneTex), anti-baculoviral IAP repeat containing 5 rabbit polyclonal antibody (GTX100052; GeneTex) and anti-CYP3A4 rabbit polyclonal antibody (GTX101236; GeneTex). The membrane was stripped and reprobed with an anti- $\beta$-actin mouse monoclonal antibody (A5441; Sigma-Aldrich, St. Louis, MO, USA), to verify loading and transfer.

Knockdown of p21 by RNA interference. Short interfering RNA (siRNA) oligonucleotides for p21 and a negative control were purchased from Invitrogen (Carlsbad, CA, USA). Three independent oligonucleotides were used for p21 siRNA as follows: a p21 siRNA1 sequence, 5'-GAU GUC CGU CAG AAC CCA UGC GGC A-3'; a p21 siRNA2 sequence, 5'-UGC CGC AUG GGU UCU GAC GGA CAU C-3'; and a p21 siRNA3 sequence, 5'-UGA GCC GCG ACU GUG AUG CGC UAA U-3'. Transfection was carried out using Lipofectamine RNAiMAX (Invitrogen) according to the manufacturer's protocol. Briefly, 60 pmol of siRNA and $10 \mu 1$ of Lipofectamine RNAiMAX were mixed in $1 \mathrm{ml}$ of RPMI medium $(10 \mathrm{nmol} / 1$ final siRNA concentration). After 20 min of incubation, the mixture was added to the cells, and these cells were plated on dishes for each assay. Cells were analyzed for all experiments at $48 \mathrm{~h}$ after transfection.

Statistical analysis. The results of the MTT assay were analyzed using the Mann-Whitney U test. All statistical analyses were conducted using StatView 5.0 software package (SAS Institute, Inc., Cary, NC, USA). A P-value of $<0.05$ was considered to be a statistically significant result.

\section{Results}

FGFR2IIIb causes reduced viability and mesenchymal markers while enhancing caspase-3 in PC-3 cells. To investigate a potential effect of FGFR2IIIb on chemosensitivity, we expressed FGFR2IIIb in PC-3 cells by transfection. Analysis of the survival of FGFR2IIIb-transfected PC-3 

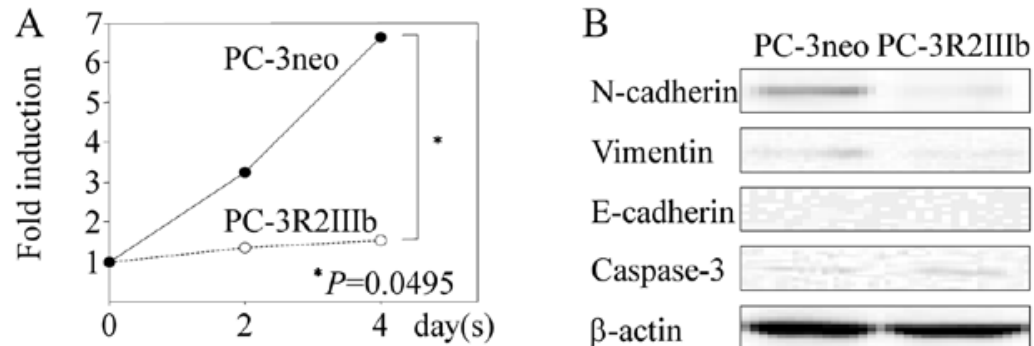

Figure 1. Effect of restoration of FGFR2IIIb on PC-3 cells. (A) FGFR2IIIb induced a decrease in cell viability. Cell viability was assessed in the FGFR2IIIbtransfected PC-3 cells (PC-3R2IIIb) and vector control-transfected cells (PC-3neo) with the MTT assay. Cultures of 1,000 PC-3 cells were incubated in OPTI-MEM1 medium containing 5\% heat-inactivated FBS. Representative results of three trials are shown. Data are presented as the mean \pm SD of the OD $570 \mathrm{~nm}$. An asterik indicates a significant decrease in cell viability relative to the PC-3 cells transfected with the vector alone (P<0.05, Mann-Whitney U test). (B) Selective decrease in expression of N-cadherin and vimentin in PC-3R2IIIb cells. Markers of epithelial-to-mesenchymal transition were analyzed by western blot analysis.

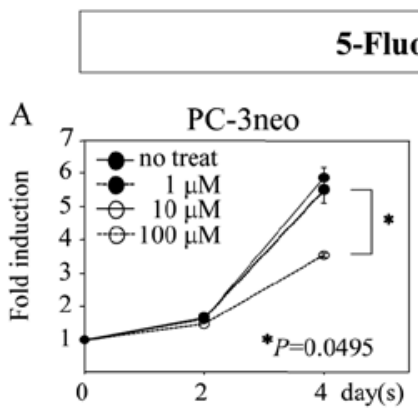

\section{-Fluorouracil}
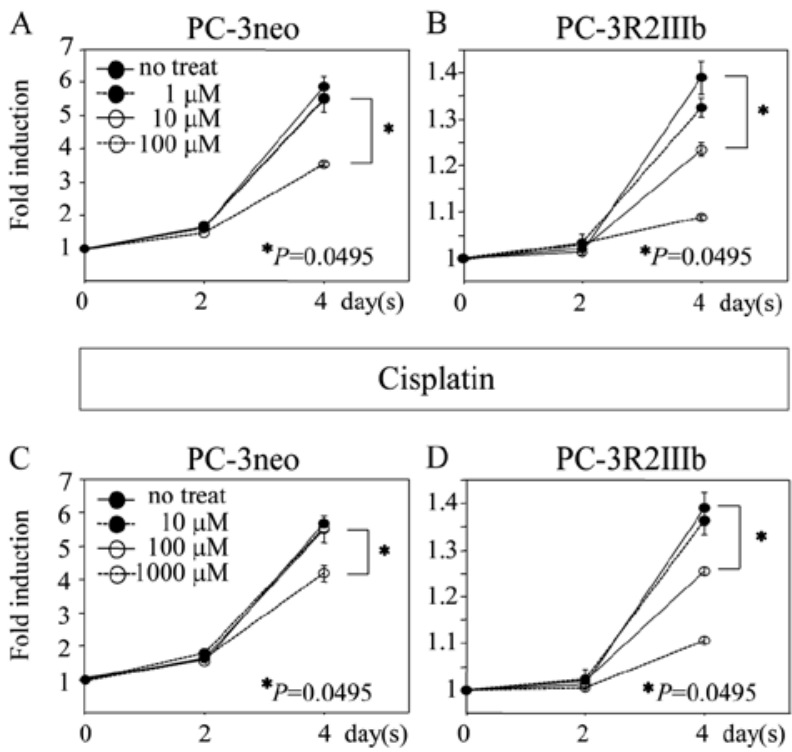

Cisplatin

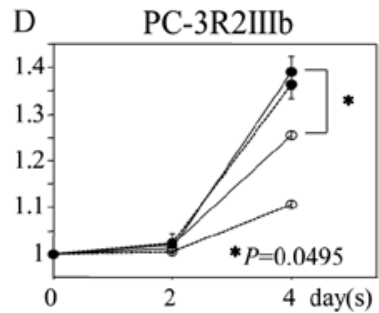

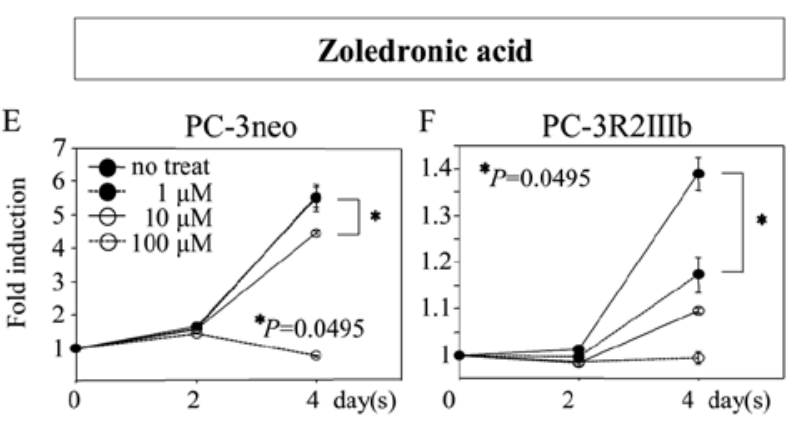

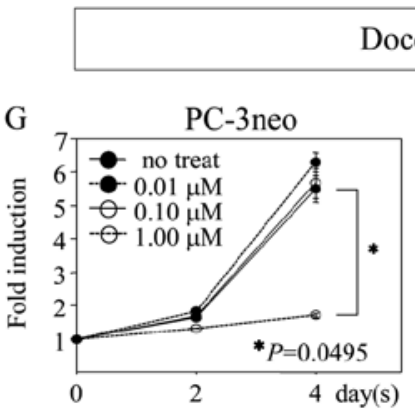

Docetaxel

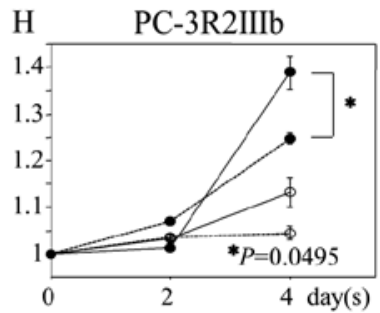

Figure 2. FGFR2IIIb increases the chemosensitivity of PC-3 cells particularly to docetaxel. Cell survival of PC-3neo and PC-3R2IIIb cells following treatment with various doses of chemotherapeutic agents over time was compared with MTT assay. The cells were treated with (A and B) 5-fluorouracil, (C and D) cisplatin, $(\mathrm{E}$ and $\mathrm{F})$ zoledronic acid and $(\mathrm{G}$ and $\mathrm{H})$ docetaxel. Representative results of three trials are shown. Data are presented as the mean $\pm \mathrm{SD}$ of the OD $570 \mathrm{~nm}$.

cells (PC-3R2IIIb) over time with the MTT assay indicated a significantly lower viability than that of the cells mock transfected with the empty transfection vector (PC-3neo) (Fig. 1A). Expression of $\mathrm{N}$-cadherin and vimentin were concurrently suppressed while that of caspase-3 was enhanced due to restoration of expression of FGFR2IIIb in PC-3 cells (Fig. 1B).

FGFR2IIIb enhances the chemosensitivity of PC-3 cells. To investigate whether restoration of FGFR2IIIb influences the anticancer effect of chemotherapeutic agents, the cell viabilities of PC-3neo and PC-3R2IIIb cells were compared after treatment with various anticancer agents (Fig. 2). Viability of cells expressing FGFR2IIIb was even lower than that of the control PC-3neo cells after treatment with all chemotherapeutic agents. The viability of the PC-3R2IIIb cells was suppressed by concentrations of cisplatin, 5-fluorouracil and zoledronic acid that were $10 \%$ that required to suppress the viability of the PC-3neo cells to a similar extent. Suppression of viability by docetaxel was even more notable. Docetaxel was effective at $1 \%$ of the concentration in the PC-3R2IIIb cells required to similarly suppress the viability of PC-3neo cells. A significant dose-dependent growth-suppressive effect was observed in the PC-3R2IIIb cells with as little as 0.01 or $0.10 \mu \mathrm{M}$ of docetaxel $(\mathrm{P}=0.045$ in $0.1 \mu \mathrm{M}$ and $\mathrm{P}=0.045$ in $0.01 \mu \mathrm{M}$, respectively). Docetaxel concurrently increased the expression of caspase-3 in a dose-dependent manner (Fig. 3). These data suggest that restoration of FGFR2IIIb enhances the chemosensitivity in prostate cancer cells through the induction of increased apoptosis.

FGFR2IIIb effect on biomarkers related to docetaxel chemosensitivity. We further examined the expression of a group of proteins related to chemosensitivity by western blot analysis. No difference in expression of clusterin, Mdm2, Bcl-2, Bcl-xL, 
A

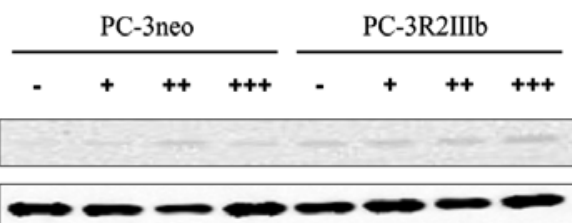

$\beta$-actin

B

Cisplatin

Caspase-3

$\beta$-actin

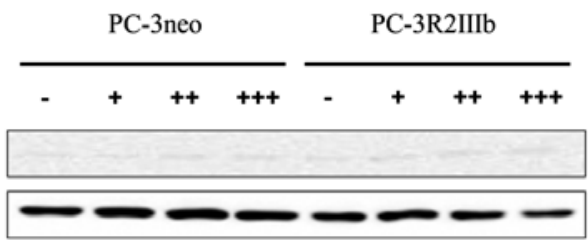

C

Zoledronic acid

Caspase-3

$\beta$-actin

D

PC-3neo

PC-3R2IIIb

Docetaxel

Caspase-3

$\beta$-actin
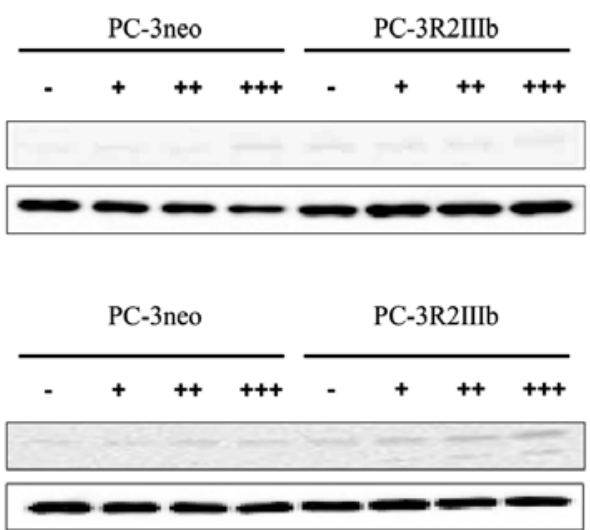

Figure 3. FGFR2IIIb enhances expression of caspase-3 in PC-3 cells exposed to chemotherapeutic agents. Expression of caspase-3 in PC-3 cells treated with anticancer drugs was analyzed by western blot analysis. (A) 5-fluorouracil, (B) cisplatin, (C) zoledronic acid and (D) docetaxel. The expression of caspase-3 was increased in a dose-dependent manner by treatment with docetaxel. A cleaved form of caspase-3 also emerged following the docetaxel treatment.
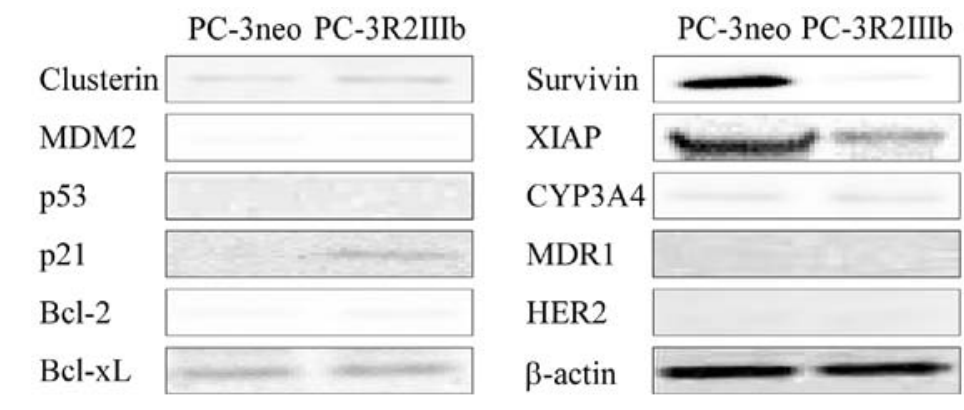

Figure 4. Effect of FGFR2IIIb restoration on proteins related to docetaxel chemosensitivity. Expression of proteins was analyzed by western blot analysis.

A

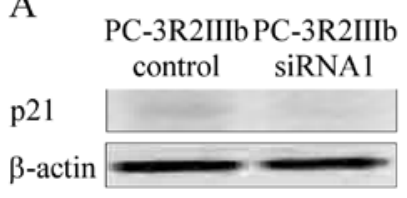

$\mathrm{B}$

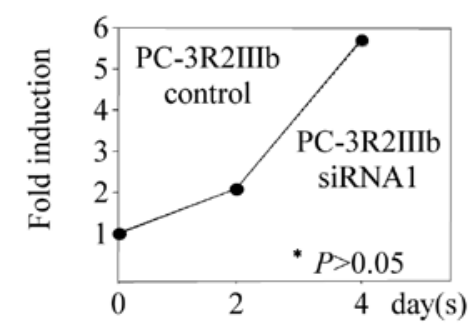

$\mathrm{C}$

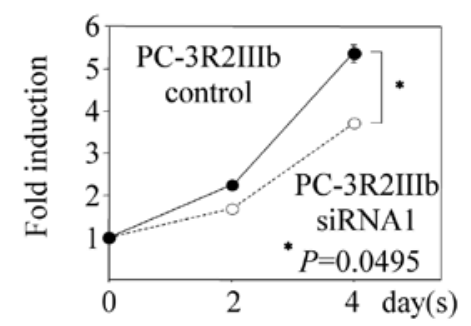

Figure 5. Effect of p21 expression on chemosensitivity. (A) Knockdown of p21. Western blot analysis of p21 expression in PC-3R2IIIb cell lines with p21 siRNA (PC-3R2IIIb siRNA1) or control scrambled siRNA (PC-3R2IIIb control) transfection. (B) Effect of p21 knockdown on cell number. (C) Effect of p21 expression on chemosensitivity. MTT assay was performed on both p21 siRNA-transfected PC-3R2IIIb cells and control scrambled siRNA-transfected PC-3R2IIIb cells after treatment with $0.1 \mu \mathrm{M}$ docetaxel.

CYP3A4, MDR1 and p53 was observed between the PC-3neo and PC-3R2IIIb cells. However, enhanced expression of p21 and suppressed expression of survivin and XIAP were evident in the PC-3R2IIIb cells compared to the PC-3neo cells (Fig. 4).

Although p21 is a cell cycle inhibitor and differentiation promoter that works in conjunction with FGFRIIIb, it has also been reported to dampen apoptosis and therefore sensitivity to cancer chemotherapy (26). To shed light on this, we used an siRNA approach to knockdown p21 and examined the effect on cell proliferation, viability and chemosensitivity. Western blotting showed that the transfected siRNA decreased the level of p21 protein (Fig. 5A) without influence on PC-3R2IIIb cell 
proliferation (Fig. 5B). However, we observed that the knockdown of p21 did contribute to a significant suppression of the viability of the PC-3R2IIIb cells in the presence of docetaxel (Fig. 5C).

\section{Discussion}

In the present study, we investigated the effect of FGFR2IIIb on the chemosensitivity of CRPC cells. To the best of our knowledge, this study is the first to report that restoration of FGFR2IIIb in CRPC cells may induce differentiation and at the same time enhance chemosensitivity through acceleration of the induction of apoptosis. It is well known that cancer cells break the basic rules of behavior by which homeostasis and normal function are maintained in normal tissues. Since cancer cells are genetically unstable to different degrees within a population, they evolve resistance when subjected to the selective pressures of irradiation or anticancer agents that target the defects in control of cell death and differentiation. The roles in homeostasis between cellular compartments in prostate tissues have been reported for FGFR2IIIb. Among prostate cancer cell lines, loss of epithelial cell FGFR2IIIb expression has been demonstrated in cell lines with high growth rates and castration-resistant cellular proliferation and other tumorigenic properties (15-21). We already showed that restoration of the FGFR2IIIb signaling pathway induces apoptosis (18) and enhances radiosensitivity in prostate cancer cells (27). On the basis of these findings, we hypothesized that the presence of a signaling pathway related to FGFR2IIIb could contribute to the efficacy of anticancer agents in PC-3 cells particularly through the induction of apoptosis.

In the present study, PC-3 cells stably transfected with FGFR2IIIb showed not only the growth-suppressive effect as demonstrated in a previous report (Fig. 1A) (18) concurrent with the decreased expression of mesenchymal markers, $\mathrm{N}$-cadherin and vimentin, compared to that in control PC-3neo cells that did not express FGFR2IIIb (Fig. 1B). These results indicate the possibility that restoration of FGFR2IIIb might reverse in part the epithelial-to-mesenchymal transition (EMT) that presumably has occurred in PC-3 cells. In essence FGFRIIIb may induce a mesenchymal-to-epithelial transition of sorts. Although EMT is a critical process in normal embryonic development, it is also associated with cancer progression and metastasis (28). Several studies have shown that EMT is associated with resistance to chemotherapy and irradiation $(28,29)$.

Restoration of FGFR2IIIb increased the sensitivity to chemotherapeutic agents in the prostate cancer cells. The efficacy of the expression of FGFR2IIIb in enhancement of chemosensitivity depended on the choice of the chemotherapeutic agent. We examined the chemosensitivity in prostate cancer cells using four drugs with different molecular and pharmacologic mechanisms of tumor suppression. The enhancement of chemosensitivity by restoration of FGFR2IIIb was most dramatic with docetaxel (Figs. 2 and 3). Docetaxel functions as an inhibitor of microtubular depolymerization (30). In addition to microtubular dynamics, multiple cellular pathways involving apoptosis, inflammation, angiogenesis, signaling intermediaries, and drug efflux pumps have been implicated in the chemosensitivity of docetaxel
(31). Previous and present data indicate that the FGFR2IIIb signaling pathway induces apoptosis and furthermore might suppress EMT or at least reverse it, which has been reported to prevent the apoptosis of cancer cells and to induce resistance to chemotherapy $(29,32)$. We then focused the investigation on changes in apoptosis-related molecules. Expression of survivin, XIAP and p21 was different between the PC-3R2IIIb and PC-3neo cells as shown in Fig. 4. Survivin and XIAP are a family of inhibitors of apoptosis proteins (IAPs) that promote cell survival by inhibiting the caspase cascade and apoptosis $(31,33)$. Survivin and XIAP are the most studied IAPs with regard to resistance to anticancer agents. Previous studies in prostate cancer cells have shown that inhibition of survivin by use of the inhibitory agent antisense and adenoviral infection enhances the sensitivity to docetaxel (34), etoposide (35) and paclitaxel (36). Furthermore, overexpression of XIAP leads to chemoresistance (37) and their inhibition improves chemosensitivity (38). From these reports, the neutralization of anti-apoptotic effects through molecular mechanisms involving survivin and XIAP might have a strong effect on sensitizing prostate cancer cells to treatment with anticancer agents. Taken together, our results point to the possibility that the induction of apoptosis through FGFR2IIIb signaling might be associated with decreased expression of survivin and XIAP. The restoration of FGFR2IIIb might enhance the sensitivity of prostate cancer cells to docetaxel by these mechanisms.

p21 is a negative regulator of cell-cycle progression in the DNA damage response, and overexpression of $\mathrm{p} 21$ can result in cell-cycle arrest in either G1, G2 or the S-phase of the cell cycle (26). The role of p21 in the effect of anticancer agents is controversial and may depend on cancer cell type and cellular context (26). Whereas several studies have shown that p21 has antiapoptotic effects and is associated with chemoresistance in cancer cells, other studies have reported p21 as a factor that enhances apoptosis (26). We found that the expression of p21 in PC-3R2IIIb was higher than that in PC-3neo cells (Fig. 5). This indicated that FGFR2IIIb enhances $\mathrm{p} 21$ and thus might contribute to the cell population growth limiting effects observed elsewhere. We then examined whether FGFR2IIIb enhanced apoptosis through induction of p21 expression or whether p21 was induced in response to an apoptotic signal transduced by overexpression of FGFR2IIIb. To clarify this point, p21 was knocked down in PC-3 cells overexpressing FGFR2IIIb. As a result, the chemosensitivity to docetaxel was enhanced by knockdown of p21 (Fig. 5). This suggested that in context of the prostate cancer cell model used here, p21 induced by FGFR2IIIb may have anti-apoptotic activity in addition to its effects on cell cycling.

Among the anticancer agents examined in the present study, FGFR2IIIb enhanced the effect of docetaxel most dramatically in the prostate cancer cells. Docetaxel is commonly used as a chemotherapy regimen for CRPC in the clinic (5). It is important to identify strategies to boost the efficacy of docetaxel and other chemotherapeutic agents as well as identify biomarkers for chemosensitivity with the aim to avoid unwarranted toxicities in patients who will not benefit from treatment. In addition to docetaxel and the agents studied here, several new chemotherapeutic agents for prostate cancer have been developed. It will be interesting to determine molecular mechanisms and associated biomarkers involved 
in the sensitivity to these agents and the potential role of the FGFR2IIIb pathway and its endpoints on the efficacy of these new strategies for CRPC.

In conclusion, FGFR2IIIb may be a key epithelial cell signaling system associated with the sensitivity of anticancer agents in CRPC cells. We propose that this may be due to its concurrent promotion of tumor cell mesenchymal-to-epithelial properties and apoptotic pathways. FGFR2IIIb, its specific pathways and endpoints may be a useful novel biomarker to predict chemosensitivity in CRPC. Clinical studies using the results here as a guide will be required to translate the manipulation of FGFR2IIIb in CRPC into clinical use.

\section{Acknowledgements}

The present study was carried out at the Analysis Center of Life Science, Hiroshima University, Japan.

\section{References}

1. Jemal A, Siegel R, Ward E, Murray T, Xu J and Thun MJ: Cancer statistics, 2007. CA Cancer J Clin 57: 43-66, 2007.

2. Jemal A, Siegel R, Ward E, et al: Cancer statistics, 2006. CA Cancer J Clin 56: 106-130, 2006.

3. Nakata S, Takahashi H, Ohtake N, Takei T and Yamanaka H: Trends and characteristics in prostate cancer mortality in Japan. Int J Urol 7: 254-257, 2000

4. Kirby R, Christmas T and Brawer M: Management of metastatic disease. In: Prostate Cancer. Hamilton L (ed). Mosby-Year Book, Inc, St. Louis, MO, pp129-141, 1996.

5. Tannock IF, de Wit R, Berry WR, et al: Docetaxel plus prednisone or mitoxantrone plus prednisone for advanced prostate cancer. N Engl J Med 351: 1502-1512, 2004.

6. Lu W, Luo Y, Kan M and McKeehan WL: Fibroblast growth factor-10. A second candidate stromal to epithelial cell andromedin in prostate. J Biol Chem 274: 12827-12834, 1999.

7. Sugimura Y, Foster BA, Hom YK, et al: Keratinocyte growth factor (KGF) can replace testosterone in the ductal branching morphogenesis of the rat ventral prostate. Int J Dev Biol 40 941-951, 1996.

8. Nakano K, Fukabori Y, Itoh N, et al: Androgen-stimulated human prostate epithelial growth mediated by stromal-derived fibroblast growth factor-10. Endocr J 46: 405-413, 1999.

9. Yan G, Fukabori Y, McBride G, Nikolaropolous $S$ and McKeehan WL: Exon switching and activation of stromal and embryonic fibroblast growth factor (FGF)-FGF receptor genes in prostate epithelial cells accompany stromal independence and malignancy. Mol Cell Biol 13: 4513-4522, 1993.

10. Naimi B, Latil A, Fournier G, Mangin P, Cussenot $O$ and Berthon P: Down-regulation of (IIIb) and (IIIc) isoforms of fibroblast growth factor receptor 2 (FGFR2) is associated with malignant progression in human prostate. Prostate 52: 245-252, 2002 .

11. Carstens RP, Eaton JV, Krigman HR, Walther PJ and GarciaBlanco MA: Alternative splicing of fibroblast growth factor receptor 2 (FGF-R2) in human prostate cancer. Oncogene 15: 3059-3065, 1997.

12. Werner S, Duan DS, de Vries C, Peters KG, Johnson DE and Williams LT: Differential splicing in the extracellular region of fibroblast growth factor receptor 1 generates receptor variants with different ligand-binding specificities. Mol Cell Biol 12: 82-88, 1992.

13. Kan M, Uematsu F, Wu X and Wang F: Directional specificity of prostate stromal to epithelial cell communication via FGF7/ FGFR2 is set by cell- and FGFR2 isoform-specific heparan sulfate. In Vitro Cell Dev Biol Anim 37: 575-577, 2001.

14. Yan G, Fukabori Y, Nikolaropoulos S, Wang $F$ and McKeehan WL: Heparin-binding keratinocyte growth factor is a candidate stromal-to-epithelial-cell andromedin. Mol Endocrinol 6: 2123-2128, 1992

15. Kwabi-Addo B, Ozen $M$ and Ittmann M: The role of fibroblast growth factors and their receptors in prostate cancer. Endocr Relat Cancer 11: 709-724, 2004.
16. Fukabori Y, Yan G, Yamanaka H and McKeehan WL: Rapid induction of keratinocyte growth factor (FGF-7) and beta-actin after exposure of prostate stromal cells to androgen. In Vitro Cell Dev Biol Anim 30A: 745-746, 1994

17. Matsubara A, Yasumoto $H$ and Usui $T$ : Hormone refractory prostate cancer and fibroblast growth factor receptor. Breast Cancer 6: 320-324, 1999.

18. Yasumoto $\mathrm{H}$, Matsubara A, Mutaguchi $\mathrm{K}$, Usui $\mathrm{T}$ and McKeehan WL: Restoration of fibroblast growth factor receptor 2 suppresses growth and tumorigenicity of malignant human prostate carcinoma PC-3 cells. Prostate 61: 236-242, 2004.

19. Matsubara A, Kan M, Feng S and McKeehan WL: Inhibition of growth of malignant rat prostate tumor cells by restoration of fibroblast growth factor receptor 2. Cancer Res 58: 1509-1514, 1998.

20. Feng S, Wang F, Matsubara A, Kan M and McKeehan WL: Fibroblast growth factor receptor 2 limits and receptor 1 accelerates tumorigenicity of prostate epithelial cells. Cancer Res 57: 5369-5378, 1997.

21. Polnaszek N, Kwabi-Addo B, Peterson LE, et al: Fibroblast growth factor 2 promotes tumor progression in an autochthonous mouse model of prostate cancer. Cancer Res 63: 5754-5760, 2003.

22. Song S, Wientjes MG, Gan Y and Au JL: Fibroblast growth factors: an epigenetic mechanism of broad spectrum resistance to anticancer drugs. Proc Natl Acad Sci USA 97: 8658-8663, 2000

23. van Bokhoven A, Varella-Garcia M, Korch C, et al: Molecular characterization of human prostate carcinoma cell lines. Prostate 57: 205-225, 2003

24. Kaighn ME, Narayan KS, Ohnuki Y, Lechner JF and Jones LW: Establishment and characterization of a human prostatic carcinoma cell line (PC-3). Invest Urol 17: 16-23, 1979.

25. Lebedeva IV, Sarkar D, Su ZZ, et al: Bcl-2 and Bcl- $\mathrm{x}_{\mathrm{L}}$ differentially protect human prostate cancer cells from induction of apoptosis by melanoma differentiation associated gene-7, $m d a-7 /$ IL-24. Oncogene 22: 8758-8773, 2003.

26. Liu S, Bishop WR and Liu M: Differential effects of cell cycle regulatory protein $\mathrm{p} 21^{\mathrm{WAF} / \mathrm{Cipl}}$ on apoptosis and sensitivity to cancer chemotherapy. Drug Resist Updat 6: 183-195, 2003.

27. Matsubara A, Teishima J, Mirkhat S, et al: Restoration of FGF receptor type 2 enhances radiosensitivity of hormone-refractory human prostate carcinoma PC-3 cells. Anticancer Res 28: 2141-2146, 2008.

28. Iwatsuki M,Mimori K, Yokobori T, et al: Epithelial-mesenchymal transition in cancer development and its clinical significance. Cancer Sci 101: 293-299, 2010.

29. Yang H, Li LW, Shi M, et al: In vivo study of breast carcinoma radiosensitization by targeting eIF4E. Biochem Biophys Res Commun 423: 878-883, 2012.

30. Pienta KJ: Preclinical mechanisms of action of docetaxel and docetaxel combinations in prostate cancer. Semin Oncol 28: 3-7, 2001.

31. Mahon KL, Henshall SM, Sutherland RL and Horvath LG: Pathways of chemotherapy resistance in castration-resistant prostate cancer. Endocr Relat Cancer 18: R103-R123, 2011.

32. Ren J, Chen Y, Song H, Chen L and Wang R: Inhibition of ZEB1 reverses EMT and chemoresistance in docetaxel-resistant human lung adenocarcinoma cell line. J Cell Biochem 114: 1395-1403, 2013.

33. Chiou SK, Jones MK and Tarnawski AS: Survivin - an antiapoptosis protein: its biological roles and implications for cancer and beyond. Med Sci Monit 9: PI25-PI29, 2003.

34. Rahman KM, Banerjee S, Ali S, et al: 3,3'-Diindolylmethane enhances taxotere-induced apoptosis in hormone-refractory prostate cancer cells through survivin down-regulation. Cancer Res 69: 4468-4475, 2009.

35. Hayashi N, Asano K, Suzuki H, et al: Adenoviral infection of survivin antisense sensitizes prostate cancer cells to etoposide in vivo. Prostate 65: 10-19, 2005.

36. Zhang M, Mukherjee N, Bermudez RS, et al: Adenovirusmediated inhibition of survivin expression sensitizes human prostate cancer cells to paclitaxel in vitro and in vivo. Prostate 64: 293-302, 2005.

37. Nomura T, Mimata H, Takeuchi Y, Yamamoto H, Miyamoto E and Nomura Y: The X-linked inhibitor of apoptosis protein inhibits taxol-induced apoptosis in LNCaP cells. Urol Res 31: 37-44, 2003.

38. Amantana A, London CA, Iversen PL and Devi GR: X-linked inhibitor of apoptosis protein inhibition induces apoptosis and enhances chemotherapy sensitivity in human prostate cancer cells. Mol Cancer Ther 3: 699-707, 2004. 